\title{
Current methods for preimplantation genetic diagnosis
}

\author{
Joanna Liss' ${ }^{1}$, Iwona Chromik ${ }^{2}$, Joanna Szczyglińska ${ }^{3}$, Monika Jagiełło², \\ Aron Łukaszuk ${ }^{1}$, Krzysztof Łukaszuk ${ }^{1-4}$ \\ 'INVICTA Fertility and Reproductive Centre, Gdansk, Poland \\ 2INVICTA Fertility and Reproductive Centre, Warsaw, Poland \\ 3INVICTA Fertility and Reproductive Centre, Wroclaw, Poland \\ ${ }^{4}$ Department of Obstetrics and Gynecological Nursing, Faculty of Health Sciences, Medical University of Gdansk, Poland
}

\begin{abstract}
Preimplantation Genetic Diagnosis (PGD) used in assisted reproduction techniques is designed to provide help for couples trying to conceive a child, as it helps deliver healthy offspring. After in vitro fertilization, material is collected from the oocyte (polar body), 3-day-old embryo, or increasingly often, from the trophectoderm of a blastocyst. Selection of the diagnostic method depends on the testing center, but methods such as aCGH (Comparative Genomic Hybridization Array) and NGS (Next-Generation Sequencing) are supposed to have the highest reliability and precision. This paper presents a review of the most important methods used in PGD, their advantages and disadvantages as well as efficacy in the procedures in which they are used.
\end{abstract}

Key words: PGD, PGS, embryo biopsy, next generation sequencing, in vitro fertilization

Ginekologia Polska 2016; 87, 7: 522-526

\section{INTRODUCTION}

In both the UK and the US, the first children born as a result of the first in vitro fertilization programs with PGD were born in 1990 [1]. Using this method, by 2004 approximately 1,000 children had been born [2], and by 2010 this number had exceeded $10,000[3,4]$. Initially, the procedure was thought to be extremely complicated and associated with a high risk of error. Currently, advances in assisted reproductive technology are made extremely rapidly, and its efficacy has been gradually increasing. At the same time, many aspects of PGD are still being discussed among specialists worldwide [5].

The procedure for preimplantation genetic diagnosis includes a sequence of activities conducted to select a genetically healthy embryo for transfer. This includes collecting material for a test (biopsy), preparation of genetic material for analysis (DNA isolation and amplification), analysis (method selection) and result preparation and issue.

Fertility clinics currently use two types of preimplantation diagnosis, depending on indications:
1. Preimplantation Genetic Diagnosis (PGD) - used to determine embryo genotype, performed in couples with genetic abnormalities such as single-gene diseases, single mutations, translocations or other gene abnormalities.

2. Preimplantation Genetic Screening (PGS) - used to determine potential aneuploidies of all 24 chromosomes. Particularly performed for patients older than 35 (AMA, Advanced Maternal Age), those with repeated implantation failures (RIF), repeated miscarriages ( $\mathrm{RM}$ ) with normal partner karyotypes, and when a severe male factor (SMF) is the cause of infertility. PGS seeks to find embryos for transfer or to select embryos with a normal karyotype [6].

\section{MATERIAL COLLECTION METHODS}

Selection of material for analysis depends on indications (through analysis of maternal material or from both parents), the extent of the predicted analysis and the technical possibilities. Material for analysis is taken from polar bodies, 
blastomeres or cells collected from the trophectoderm of blastocysts [4]. Recently, there have been reports on the possibility of collecting material from fluid in the blastocyst cavity and blastomeres from a morula stage embryo, after decompaction $[7,8]$.

\section{Diagnostic methods used in PGD/PGS}

Pursuant to reports by the ESHRE PGD Consortium (European Society of Human Reproduction and Embryology), in the period 1997-2007, 27,000 cycles focused on preimplantation diagnosis were performed: $61 \%$ were screening tests for aneuploidy; $17 \%$ were diagnoses of single-gene diseases; $16 \%$ were for chromosomal abnormalities; $4 \%$ were for chromosome $X$-linked diseases, and $2 \%$ for sex selection (Figure 1). Current methods of PGD/PGS diagnosis use fluorescent in situ hybridization (FISH), comparative genomic hybridization (CGH), single nucleotide polymorphism (SNP) analysis, and the latest method of next generation sequencing (NGS). Direct methods supporting the diagnostic process include, among others, polymerase chain

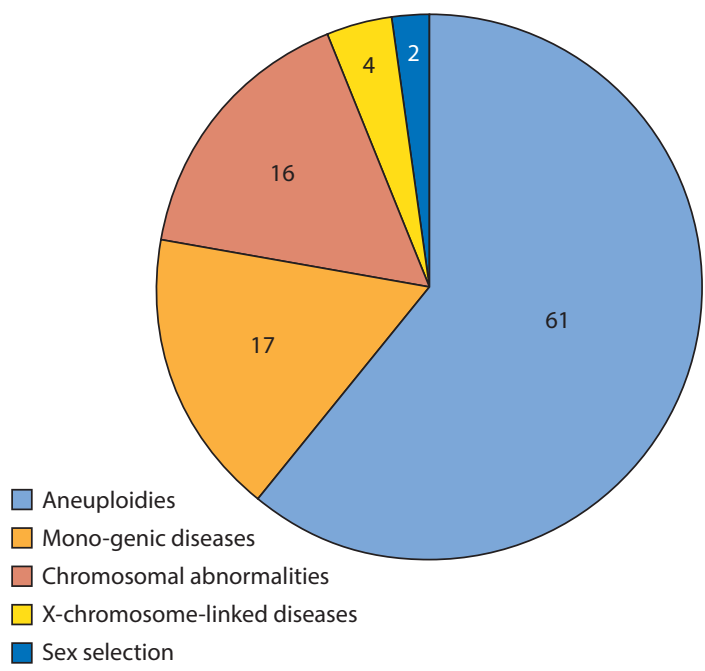

Figure 1. Indications for preimplantation diagnosis reaction (PCR) and whole genome amplification (WGA) [9-11]. Table 1 presents some examples of applications for these techniques [9].

\section{Fluorescent In Situ Hybridization (FISH)}

FISH is one of the oldest methods used in preimplantation diagnosis and was used for the first time in 1993 by Schrurs et al. to determine aneuploidy [12]. Further advances included its use in determining chromosomal translocations by Munne et al. [13].

FISH involves identification of chromosomes or their fragments with fluorescently labelled molecular probes. Probes are complementary to specific DNA regions that are subject to hybridization under specific conditions, and the result of this process can be observed as fluorescent spots under a fluorescent microscope. FISH diagnosis is performed using interphase nuclei. After an embryo biopsy blastomere (or two) is placed in a hypotonic solution, it is then digested and fixed with methanol and acetic acid [14].

It is not possible to test a whole panel of 24 chromosomes during one test, as it is only feasible to use 5-9 probes at most, for 2-3 rounds of hybridization. Therefore, PGS-FISH diagnosis is limited to the most common abnormalities involving chromosomes 13, 15-18, 21, 22, X and Y [11]. FISH diagnosis is also associated with a number of doubts regarding result reliability. Due to such problems as inappropriate blastomere fixation, probe attachment and washing away performed many times, as well as artefacting (poor/additional or completely nonspecific signals), this technique has to be passed over as imprecise and associated with a high risk of error. In 2007, a study was conducted on 206 patients subject to preimplantation diagnosis with FISH, and 202 patients in the control group, aged 35-41 years. It revealed a significantly lower rate of pregnancies in the first group (25\%) compared to the control group (37\%) [15]. Some of FISH's disadvantages were mentioned earlier, but it is also less than perfect in its need for material from 3-day-old embryos, which can disturb their further developmental

\section{Table 1. Examples of applications of PGD/PGS techniques}

\begin{tabular}{|l|c|c|c|c|}
\hline Indications & FISH & aCGH & SNP array & NGS \\
\hline Sex selection & YES & YES & YES & YES \\
\hline Screening tests for aneuploidy & YES/Specific locus & YES & YES & YES \\
\hline Aberrations associated with a changed number of DNA copies & YES/Specific locus & YES & YES & YES \\
\hline Chromosomal rearrangements (translocations) & YES/Specific locus & YES* & YES* & YES* \\
\hline Single-gene mutations & NO & NO & YES & YES \\
\hline De novo mutations & NO & NO & NO & YES \\
\hline Mitochondrial mutations & NO & NO & NO & YES \\
\hline
\end{tabular}

${ }^{*}$ changes in fragments > $6 \mathrm{MB}$; aCGH — array comparative genomic hybridization; FISH — fluorescent in situ hybridization; NGS — next generation sequencing; SNP - single nucleotide polymorphism 
potential, and because the number of analyzed chromosomes is limited. In this regard, a positive FISH result does not indicate a 'healthy' embryo $[14,15]$.

FISH is also used to determine translocations. However, this field of study has now been successfully replaced by methods that are more reliable and precise, such as SNP [16] and NGS [17].

\section{Comparative Genomic Hybridization (CGH)}

Comparative genomic hybridization was introduced in molecular cytogenetics in 1992 by Kalioniemi et al. [18]. This method is used to detect duplication or deletion of chromosome fragments without the need for culturing cells. The method is based on using standard (normal) DNA labelled with a fluorescent dye, and tested DNA (from a patient) that is also labelled with a fluorochrome. Both genomes are placed on a slide or metaphase plate. In this way, two genomes coded with different colors and cut enzymatically into small fragments reorganize on chromosomes using the rule of complementarity and competition for hybridization sites. All quantitative differences between them are visible as a predominance of one color over the other $[11,15]$. The main disadvantage of the classic CGH method is its low resolution, which averages $10 \mathrm{Mbp}$, and so an improved version, known as array CGH $(\mathrm{aCGH})$, is used in preimplantation diagnosis.

Array CGH is considered to be precise ( $2.9 \%$ no errors), highly specific (1.9\% errors) and not time-consuming (analysis of up to $24 \mathrm{~h}$ ). Although it is not possible to use aCGH for haploid or polyploid embryo, low-grade mosaicism and single mutations, research has proved that a significant majority of embryos with such dysfunctions also have additional disorders that can be detected with aCGH (such as aneuploidy), and which are not present in approximately $0.2 \%$ of cases $[19,20]$.

In 2011, Alfarawati et al. were one of the first to publish a report on delivery of the first healthy child subjected to aCGH-based PGD at the embryonal stage, for unbalanced chromosome configuration associated with a balanced translocation in one of its parents [21]. Schoolcraft et al. demonstrated a statistically significant increase in the rate of embryo implantations from $46.5 \%$ to $72.2 \%$ ( $p<0.001)$ for embryos that had been biopsied at the blastocyst stage, frozen and verified with CGH-based PGS [22].

Chromosomal translocations are another application for PGD/CGH. Reciprocal translocations involve the exchange of segments between non-homologous chromosomes. They can be balanced - there are no abnormalities — or unbalanced, where there is an excess or lack of some chromosome material. Robertsonian translocations result when the long arms of acrocentric chromosomes fuse (13-15, 21, 22), and the short arms of these chromosomes are lost. As they do not carry any significant genetic information, an abnormal genotype is not formed. Being a translocation carrier increases the risk of failure by even $85 \%$, which is due to the fact that the carrier produces gametes with an unbalanced karyotype. Therefore, PGD should include detection of translocations as well as screening tests of chromosomes with regard to numeric abnormalities. Array CGH can successfully be used in PGS, but translocation detection is associated with limitations as it is possible to detect translocated fragments that are not smaller than $6 \mathrm{Mb}$ [20]. However, in their study Colls et al. demonstrated that in diagnosis of structural chromosomal abnormalities, at an early embryonic stage it is more effective to use aCGH than FISH; the precision of translocation diagnosis was assessed on detection of at least three translocated chromosomal fragments. This study also demonstrated the necessity of PGS in selecting a healthy embryo, as $26.4 \%$ of normal or balanced embryos demonstrated aneuploidy [23]. This thesis was also confirmed in 2011 studies by Gutierrez-Mateo et al. — in a group of 81 embryos diagnosed as normal or balanced with regard to translocations, 51 had chromosomal aneuploidy that disqualified embryos from transfer [24]. Similar studies with comparable results were also performed by Fiorentino et al. [25] and Pujol et al. [26].

Comparative genomic hybridization is the most common method used by research centers and there is a large amount of data confirming its efficacy, but according to ESHRE guidelines, it is still necessary to validate this method before it can be introduced into routine diagnosis [27].

\section{Single nucleotide polymorphism (SNP) analysis}

Single nucleotide polymorphisms are places in a genome where one nucleotide in a specific locus is different from the others in the population. Methods using SNP markers utilize platforms/plates allowing for determination of thousands or millions of SNPs in a human population during a single DNA analysis. There are a large number of SNP methods based on hybridization, starter elongation, ligation or so-called invasive rupture, all of which favor the accessibility of this method [28].

In 2010, Treff et al. designed and validated a method to screen 24 chromosomes for aneuploidy that was based on SNP identification [29]. Since then, many centers have used this method for PGS and obtained satisfactory results.

Schoolcraft et al. considered three variables in PGS diagnosis: blastocyst biopsy, embryo vitrification and chromosome screening with SNP array. After analysis, $47.4 \%$ of blastocysts (356/751) were diagnosed as euploid and qualified for transfer. Results were not obtained in 4.5\%. Biochemical pregnancies were obtained in 87\% (87/100), clinical pregnancies with fetal heart beats in $73 \%$ (73/100). Miscarriage was observed in two cases $-2.7 \%$. As a result of the combined 
activities of a research team, a high implantation rate and low miscarriage rate was observed in infertile couples [30]. Studies with SNP platforms were also conducted on diagnosis of balanced, unbalanced and Robertsonian translocations, and good outcomes were observed [16].

\section{Next Generation Sequencing (NGS)}

Next Generation Sequencing belongs to the group of Massively Parallel Sequencing (MPS) methods that allow for parallel processing of an extremely large number of nucleic acid molecules. As a result of sequencing on a microspace scale (e.g. reaction vessels with a diameter of 3 micrometers), it has been possible to drastically increase the amount of information collected during one test, up to an entire human genome. NGS has revolutionized sequencing even more than microarrays revolutionized CGH. Within subsequent years of its existence, the costs of equipment, disposables and chemicals have fallen, which has increased the accessibility and popularity of its use in research and medical fields.

Currently, more than ten NGS platforms are available. Post-Light NGS, where semiconductor chips are used instead of laser-optic systems and color reactions allowing for translation of genetic information directly into binary signals, has to be mentioned. In preimplantation diagnosis NGS has become a better alternative than aCGH. NGS can be used in fresh and frozen cycles, and it is possible to analyze almost all types of genetic variability. NGS is also the only method that allows for analysis for aneuploidy or translocation of all chromosomes and mutations responsible for any single-gene disease, using one biopsy and one process.

Using next generation sequencing it is possible to study an entire genome with regard to different abnormalities such as aneuploidy, single-gene mutations or de novo mutations. It is quite costly to equip a laboratory with all of the equipment necessary to perform NGS procedures, but this technology allows for performance of analyses of many samples at the same time, thereby reducing the costs of a single analysis.

When NGS is used in PGD it is necessary to isolate the DNA and amplify the whole genome (from a single cell or several cells). Then, the material is cut into small DNA fragments (of 100-200 base pairs) and placed on a $2 \times 2 \mathrm{~cm}$ 'chip'. It seems incredible to place a hundred thousand DNA fragments from different patients on a single chip, but this is possible thanks to DNA barcoding [31]. A sequence of each fragment is then compared to a reference sequence. The results are prepared by a computer.

Łukaszuk et al. used NGS to screen 24 chromosomes for aneuploidy in PGD [32]. This study included 45 patients with a history of recurrent miscarriages. A control group included 53 patients, matched by age, reason for infertility, AMH levels and number of antral follicles. In a group of 252 studied em- bryos, 142 demonstrated aneuploidy, 21 were considered to be non-diagnostic, and 89 had a normal karyotype. 65 transfers were performed among 89 normal embryos. There were 89 transfers in the control group. With regard to the final outcome, the pregnancy rate in the study group was twice that of the control group ( $84.4 \%$ vs. $41.5 \%$, respectively). An analysis made using NGS was also performed for a couple carrying a Robertsonian translocation, and the whole process of diagnosing the translocation and aneuploidy of 24 chromosomes resulted in implantation of a healthy embryo and normal pregnancy development [17]. Similar findings were observed by Fiorentino et al. [33], Tan et al. [34] and Treff et al. who successfully used next generation sequencing to identify monogenic diseases [35].

Currently, NGS is thought to be a future and target technology for PGD/PGS. As a gold standard for genetics sequencing, it allows for the almost $100 \%$ study of an entire gene/genome, and so it is possible to obtain a 'perfect' analytical result.

\section{SUMMARY}

Currently, it is known that more than $50 \%$ of embryos obtained under in vitro conditions have genetic abnormalities. This number increases to $80 \%$ in patients older than 40 . In the majority of abnormal embryos, development is arrested between day 3 and day 5, but the remaining embryos develop until the blastocyst stage, and are then transferred to the uterus or frozen and used in the next cycle. This rate of genetic abnormality is thought to be present not only in cycles stimulated with in vitro fertilization, but also in cycles stimulated with a natural approach to conception (i.e. insemination) [20].

Preimplantation diagnosis combined with modern technology represents a new era of infertility treatment for couples with a chance of healthy pregnancy as early as within one cycle. It saves time, and the patient's ovarian reserve. Additionally, it avoids the mental burden associated with repeated miscarriages and failures, as well as saving wear and tear on the woman's body and reproductive organs.

The following conclusions can be drawn based on discussions and research papers presented by scientists worldwide:

- Fluorescent in situ hybridization has been replaced by more reliable technologies, such as aCGH and NGS, where each chromosome can be tested for chromosomal abnormalities;

- Biopsy of 3-day-old embryos is being less and less frequently performed and is in fact being replaced by biopsy of the trophectoderm of the blastocyst; it is thought that the embryo blastomere is not representative of the entire whole genome due to high levels of mosaicism, and that results are often 
non-diagnostic due to the low amount of material available for analysis. Thanks to the trophectoderm it is possible to obtain several cells with a lower chance of mosaicism, consequently increasing the chances of obtaining reliable results;

- Embryos that have been successfully biopsied and frozen can be transferred in the next cycle; according to various studies, freezing does not have a significant effect on further embryo development or implantation. It has also been suggested that primed endometrium favors better implantation in the next cycle;

- PGD/PGS technology seems to be more and more associated with routine use of comparative genomic hybridization and next generation sequencing, and these methods are recommended by ESHRE. However, they have to be validated for introduction to routine diagnostics in fertility clinics.

There are ongoing multicenter, randomized studies aimed at optimizing the strategy for PGD to achieve the highest efficacy in infertility treatment, as well as the highest reliability.

\section{REFERENCES}

1. Handyside $A H$, Kontogianni EH, Hardy $K$, [et al.]. Pregnancies from biopsied human preimplantation embryos sexed by Y-specific DNA amplification. Nature. 1990, 344, 768-770.

2. Verlinsky Y, Cohen J, Munné S, [et al.]. Over a decade of experience with preimplantation genetic diagnosis: a multicenter report. Fertility and Sterility. 2004, 82, 292-294.

3. Simpson JL. Preimplantation genetic diagnosis at 20 years. Prenat Diagn. 2010, 30, 682-695

4. Coco R. Reprogenetics: Preimplantational genetic diagnosis. Genetic and Molecular Biology. 2014, 37, 271-284.

5. Brezina R. Preimplantation Genetic Testing in the 21st Century: Uncharted Territory. Clinical Medicine Insights: Reproductive Health. 2013, 7, 17-21.

6. Harper JC, Geraedts J, Borry P. Current issues in medically assisted reproduction and genetics in Europe: research, clinical practice, ethics, legal issues and policy. Human Reproduction. 2014, 29, 1603-1609.

7. Gianaroli L, Magli MC, Pomante A, [et. al.]. Blastocentesis: a source of DNA for preimplantation genetic testing. Fertility and Sterility. 2014, 102, 1692-1699.

8. Milachich T. New advances of preimplantation and prenatal genetic screening and noninvasive testing as a potential predictor of health status of babies. BioMed Research International. 2014, 306505, 8.

9. Niels A, Esteki MZ, Vermeesch JR, [et.al.]. Preimplantation genetic diagnosis guided by single-cell genomics. Genome Medicine. 2013, 5, 71.

10. Harper JC, Wilton L, Traeger-Synodinos J, [et al.] The ESHRE PGD Consortium: 10 years of data collection. Hum Reprod. 2012, 18, 234-247.

11. Wu MY, Chao KH, Chen CD, [et al.] Current status of comprehensive chromosome screening for elective single-embryo transfer. Obstet Gynecol Int. 2014, 2014:581783.

12. Schrurs BM, Winston RM, Handyside AH. Preimplantation diagnosis of aneuploidy using fluorescent in-situ hybridization: evaluation using a chromosome 18-specific probe. Hum Reprod. 1993, 8, 296-301.

13. Munné $S$, Scott $R$, Sable $D$, Cohen J. First pregnancies after preconception diagnosis of translocations of maternal origin. Fertil Steril. 1998, 69(4), 675-681.
14. Eduardo CL, Marleen MJ, Carl BB. Preimplantation Genetic Testing: Current Status and Future Prospects. Ed. Shevach Friedler. In vitro fertilization: Innovative clinical and laboratory aspects. InTech 2012, 137-156.

15. Mastenbroek S, Twisk M, van Echten-Arends J, [et .al.]. In vitro fertilization with preimplantation genetic screening. NEngl J Med. 2007, 357, 9-17.

16. Tan $Y Q$, Tan $K$, Zhang SP, [et al.]. Single-nucleotide polymorphism microarray-based preimplantation genetic diagnosis is likely to improve the clinical outcome for translocation carriers. Hum Reprod. 2013, 28, 2581-2592.

17. Łukaszuk K, Pukszta S, Ochman K, [et al.]. Narodziny zdrowego dziecka u pary z nosicielstwem translokacji Robertsonowskiej po zastosowaniu Genetycznej Diagnostyki Przedimplantacyjnej (PGD) z Sekwencjonowaniem Następnej Generacji (NGS). ARTNewsletter, 2014, 2, 31-33.

18. Kallioniemi A, Kallioniemi OP, Sudar D, [et al.] Comparative genomic hybridization for molecular cytogenetic analysis of solid tumors. D. Science. 1992, 258, 818-821.

19. Gutiérrez-Mateo C, Colls P, Sánchez-García J, [et al.] Validation of microarray comparative genomic hybridization for comprehensive chromosome analysis of embryos. Fertil Steril. 2011, 95, 953-958.

20. Munne S. Preimplantation Genetic Diagnosis for aneuploidy and translocations using array comparative genomic hybridization. Current Genomics, 2012, 463-470,

21. Alfarawati S, Fragouli E, Colls P, Wells D. First births after preimplantation genetic diagnosis of structural chromosome abnormalities using comparative genomic hybridization and microarray analysis. Hum Reprod. 2011, 26, 1560-1574.

22. Schoolcraft WB, Fragouli E, Stevens J, [et al.] Clinical application of comprehensive chromosomal screening at the blastocyst stage. Fertil Steril. 2010, 94, 1700-1706.

23. Colls P, Escudero T, Fischer J, [et al.] Validation of array comparative genome hybridization for diagnosis of translocations in preimplantation human embryos. Reprod Biomed. 2012, 24, 621-629.

24. Gutiérrez-Mateo C, Colls $P$, Sánchez-García J, [et al.]. Validation of microarray comparative genomic hybridization for comprehensive chromosome analysis of embryos. Fertil Steril. 2011, 95, 953-958.

25. Fiorentino F, Spizzichino L, Bono S, [et al.]. PGD for reciprocal and Robertsonian translocations using array comparative genomic hybridization. Pantos K Hum Reprod. 2011, 26, 1925-1935.

26. Pujol A, Benet J, Staessen C, [et al.] The importance of aneuploidy screening in reciprocal translocation carriers J Reproduction. 2006, 131, 1025-1035.

27. Harper J, Coonen E, De Rycke M, [et al.].What next for preimplantation genetic screening (PGS)? A position statement from the ESHRE PGD Consortium Steering Committee. Hum Reprod. 2010, 25, 821-823.

28. Sobrino B, Brión M, Carracedo A. SNPs in forensic genetics: a review on SNP typing methodologies. Forensic Sci Int. 2005, 154, 181-194.

29. Treff NR, Su J, Tao X, [et al.] Accurate single cell 24 chromosome aneuploidy screening using whole genome amplification and single nucleotide polymorphism microarrays. Fertil Steril. 2010, 94, 2017-2021.

30. Schoolcraft W, Treff N, Stevens J. Live birth outcome with trophoectoderm biopsy, blastocyst vitrification and single-nucleotide polymorphism microarray-based comprehensive chromosome screening in infertile patients. Fertil Steril. 2011, 96, 638-640.

31. Knapp M, Stiller M, Meyer M. Generating barcoded libraries for multiplex high-throughput sequencing. Methods Mol Biol. 2012, 840, 155-170.

32. Łukaszuk K, Pukszta S, Wells D, [et al.]. Routine use of next-generation sequencing for preimplantation genetic diagnosis of blastomeres obtained from embryos on day 3 in fresh in vitro fertilization cycles. J Fertil Steril. 2015, Jan 23.

33. Fiorentino F, Bono S, Biricik A, [et al.]. Application of next-generation sequencing technology for comprehensive aneuploidy screening of blastocysts in clinical preimplantation genetic screening cycles. Hum Reprod. 2014, 29, 2802-2813.

34. Tan $Y$, Yin $X$, Zhang $S$, [et al.]. Clinical outcome of preimplantation genetic diagnosis and screening using next generation sequencing. Gigascience. 2014, 3, 30.

35. Treff NR, Fedick A, Tao X, [et al.]. Evaluation of targeted next-generation sequencing-based preimplantation genetic diagnosis of monogenic disease. Fertil Steril. 2013, 99, 1377-1384. 